\title{
Increased erythrocyte aggregation as an indicator for an aggressive clinical course in Behçet's disease: a prospective study
}

\author{
Halûk Demiroğlu, Şuayib Yalçın, Yahya Büyükaşık, Osman İlhami Özcebe, Semra Dündar
}

Departments of

Haematology

H Demiroğlu

Y Büyükaşık

O İ Özcebe

$S$ Dündar

and Medical Oncology Ş Yalçın

Hacettepe University

Medical School,

Ankara,Turkey

Correspondence to:

Dr H Demiroğlu, Hoșdere

Cad 80/19, Yukarı Ayranc1

06550, Ankara, Turkey.

Accepted for publication 23 July 1998

\begin{abstract}
Objective-Changes in blood rheology, especially increased erythrocyte aggregation (EA) might play an important part in the development of arterial and venous thrombotic lesions. A prospective study was designed to evaluate EA in patients with Behçet's disease (BD) and to see if this parameter is predictive for the future development of vascular complications, such as deep vein thrombosis of various organ systems and uveitis.
\end{abstract}

Methods-EA was measured by a photometric Myrenne aggregometer in 38 patients with $\mathrm{BD}$ at the time of initial diagnosis and in 40 age and sex matched healthy controls (HC).

Results-During a median follow up period of 13.5 months, 13 patients developed vascular-ocular complications (eight deep vein thrombosis, nine uveitis, and four both deep vein thrombosis and uveitis). Patients were further divided into two groups: BD-a with mucocutaneous symptoms and arthritis only; BD-b with associated vascular-ocular complications. EA values at high shear rate $(M)$ and at low shear rate (M1) were compared among the groups.

Conclusion-EA values at $M$ and $M 1$ were significantly higher in $\mathrm{BD}-\mathrm{b}$ than $\mathrm{BD}-\mathrm{a}$ and HC $(p<0.001)$. These results suggest that determination of EA rates might be useful to identify subgroups who are likely candidates for developing vascular-ocular complications in $\mathrm{BD}$ and management of factors known to affect blood rheology might be beneficial.

(Ann Rheum Dis 1998;57:694-696)

Table 1 Characteristics and initial findings of $B D$ patients and $H C$

\begin{tabular}{lllll}
\hline & BD patients & $H C$ & $B D-a$ & $B D-b$ \\
\hline Age (y) & & & & \\
$\quad$ median & 27 & 28 & 28 & 24 \\
$\quad$ range & $21-39$ & $20-40$ & $22-39$ & $21-35$ \\
Sex (M/F) & $23 / 15$ & $24 / 16$ & $15 / 10$ & $8 / 5$ \\
Oral aphthae (n) & 38 & & 25 & 13 \\
Genital ulcers (n) & 35 & & 23 & 12 \\
Skin lesions (n) & 33 & & 21 & 12 \\
Positive pathergy test (n) & 24 & & 16 & 8 \\
Arthritis (n) & 15 & $43.9(0.46)$ & $43.5(0.56)$ & $43.16(0.9)$ \\
Packed cell volume (\%) & $43.4(0.48)$ & $187.4(2.7)$ & $186.8(3.6)$ & $183.9(6.8)$ \\
Cholesterol (mg/dl) & $185.8(3.3)$ & $235.8(4.8)$ & $245(6.3)$ & $255.3(9)$ \\
Fibrinogen (mg/dl) & $248.5(5.1)$ & & $31.1(3.8)$ & $30.8(6)$ \\
ESR (mm 1st h) & $31(3.2)$ & & $1.23(0.25)$ & $1.18(0.38)$ \\
CRP (mg/dl) & $1.22(0.2)$ & & & \\
\end{tabular}

${ }^{\star}$ Includes folliculitis, papulopustular and acneiform lesions and erythema nodosum. Data shown as numbers, percentages, and mean (SD).
Behçet's disease (BD) is a multisystem inflammatory disorder characterised by recurrent oral and genital ulceration, uveitis, skin lesions, thrombosis of deep veins, arterial occlusion and/or aneurysms, arthritis, and central nervous system involvement (CNS). ${ }^{1}$ While $\mathrm{BD}$ runs an indolent course in some patients with mucocutaneous manifestations dominating the clinical picture, on the other side of the spectrum, serious vascular complications such as uveitis and vascular occlusive disease may lead to great morbidity and even mortality. ${ }^{2}$ The exact cause of these vascular problems is not precisely known. Anticardiolipin antibodies and increased lipoprotein-a concentrations leading to a decreased fibrinolytic activity have been reported as causative factors for a thrombotic tendency in $\mathrm{BD} .{ }^{3}{ }^{4}$ It is well known that changes in haemorheological factors, especially increased erythrocyte aggregation (EA) might play an important part in the development of vascular thrombotic lesions both in arterial and venous systems. ${ }^{56}$ Vascular lesions are a characteristic feature of $\mathrm{BD}$, however, there is no simple determinant in whom a serious vascular complication will develop during the course of the disease. We designed a prospective study to investigate if EA was increased in patients with BD and to show if increased EA had a predictive value for future vascular complications.

\section{Methods}

PATIENTS AND CONTROLS

Thirty eight patients with BD and 40 age and sex matched healthy controls (HC) were enrolled into the study. Diagnosis of BD was based on the criteria proposed by the International Study Group for Behçet's Disease. ${ }^{7} \mathrm{BD}$ patients were newly diagnosed and none of them had serious vascular complications such as uveitis, venous or arterial thrombosis of various systems and CNS disease at the time of enrolment. Table 1 shows the clinical and laboratory features of $\mathrm{BD}$ patients and $\mathrm{HC}$. BD patients were taking colchicine daily and benzathine penicilline once every three weeks. When vascular thrombosis developed, we began short-term anticoagulation followed by dipyridamole. In the case of eye involvement, topical corticosteroids together with systemic corticosteroids were used until inflammation subsided; then interferon was begun.

BD patients were followed up for a median of 13.5 months (range 6-24). During the follow up period, 13 patients developed vascular-ocular problems; eight patients devel- 


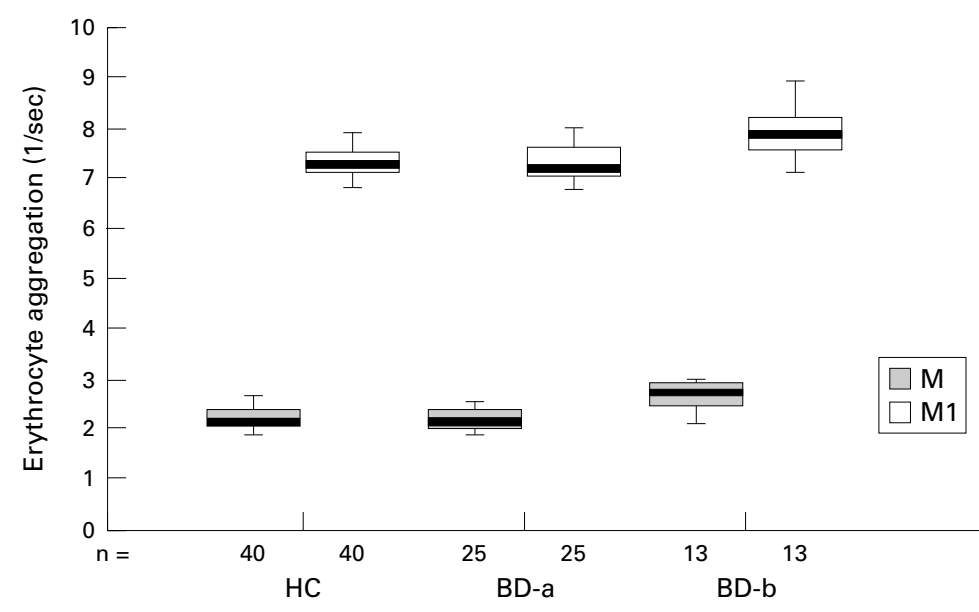

Figure 1 EA values of each group at $M$ and $M 1$ ( $n=$ number of subjects in each group).

oped deep vein thrombosis of various organ systems, nine patients developed uveitis, and four patients developed both deep vein thrombosis and uveitis. We further divided patients into two groups according to these vascularocular complications: BD-a $(\mathrm{n}=25)$ with mucocutaneous symptoms and arthritis only; $\mathrm{BD}-\mathrm{b}(\mathrm{n}=13)$ with associated vascular-ocular complications (table 1 ).

\section{LABORATORY STUDIES}

EA was studied by a photometric rheoscope (Myrenne aggregometer), which measures EA in $20 \mu \mathrm{l}$ of fasting venous blood anticoagulated with EDTA. Measurements were made at packed cell volume adjusted to $45 \%$ by removing or adding autologous plasma, after shearing at $600 / \mathrm{sec}$, at stasis (high shear rate, $M$ ) and at $3 / \mathrm{sec}$ (low shear rate, M1). ${ }^{8}$ All other parameters (packed cell volume, cholesterol, fibrinogen, erythrocyte sedimentation rate (ESR), and $C$ reactive protein $(\mathrm{CRP})$ ) were tested by routine methods.

STATISTICAL METHODS

Mann-Whitney U test, Kruskal-Wallis test, and analysis of variance were used for comparisons among $\mathrm{BD}-\mathrm{a}, \mathrm{BD}-\mathrm{b}$, and $\mathrm{HC}$ groups where appropriate. Multivariate analysis was performed by using a multiple regression model. All values are expressed as mean (SD). A value of $\mathrm{p}<0.05$ was accepted as statistically significant.

\section{Results}

No difference was found among the study groups $\mathrm{BD}-\mathrm{a}, \mathrm{BD}-\mathrm{b}$ and $\mathrm{HC}$ in terms of age, sex, packed cell volume, cholesterol, and fibrinogen values (table $1, \mathrm{p}>0.05$ ).

ESR values in BD-a and BD-b were similar as well $(p>0.05)$. When we took into consideration the patients with arthritis, we saw that ESR value of these patients was 52.2 (3.4) and those without arthritis was $17.21(1.23)(\mathrm{p}<0.001)$. There was no correlation between ESR and EA values at $M$ and $M 1$. CRP values in BD-a and $\mathrm{BD}-\mathrm{b}$ were similar, too $(\mathrm{p}>0.05)$. Likewise, CRP was higher in patients with arthritis than those without (2.55 (0.27) $v 0.34$ (0.04), $\mathrm{p}<0.001)$. There was no correlation between $\mathrm{CRP}$ and $\mathrm{EA}$ at $\mathrm{M}$ and $\mathrm{M} 1$, either.
At the time of initial diagnosis, EA values at $\mathrm{M}$ in BD-a, BD-b and HC were as follows: 2.2 (0.04), 2.7 (0.07), and 2.25 (0.03), respectively (fig 1). The difference was significantly higher in BD-b than BD-a and HC $(\mathrm{p}<0.001)$, whereas there was no statistically significant difference between $\mathrm{BD}-\mathrm{a}$ and $\mathrm{HC}$ groups $(\mathrm{p}>0.05)$. EA values at $\mathrm{M} 1$ in $\mathrm{BD}-\mathrm{a}, \mathrm{BD}-\mathrm{b}$, and HC were as follows: $7.32(0.07), 8(0.17)$, and 7.33 (0.04), respectively. Although there was no statistically significant difference between BD-a and HC, M1 was significantly higher in BD-b than BD-a and HC $(p<0.001)$. After the vascular-ocular attack, as patients were taking dipyridamole or interferon treatment, EA was reassessed as post-treatment $M$ (post- $M$ ) and post-M1. In BD-a and BD-b patients, post-M values were as follows: $2.21(0.06)$ and 2.55 (0.06), respectively, and post-M1 as: 7.26 (0.07) and $7.66(0.16)$, respectively. Although post-M and post-M1 were significantly lower than $\mathrm{M}$ and $\mathrm{M} 1$, respectively, in $\mathrm{BD}-\mathrm{b}$ $(p<0.01)$, these values were still higher than $M$ and $\mathrm{M1}$ values of $\mathrm{BD}-\mathrm{a}$ patients and $\mathrm{HC}$ $(\mathrm{p}<0.05)$.

In multivariate analysis for the determination of factors influencing EA rates at $M$ and M1, only vascular-ocular involvement was found to be significant, whereas age, sex, packed cell volume, cholesterol, fibrinogen, ESR, and CRP values had no significant effects. To demonstrate the prognostic value of erythrocyte aggregability, we calculated the relative risk ratio. The relative risk of vascularocular attack in BD patients with an EA value higher than HC was 12 for M and 5.5 for M1.

\section{Discussion}

$\mathrm{BD}$ is highly variable in its presentation and clinical course and there exists a marked individual variability. ${ }^{9}$ Not all patients with $\mathrm{BD}$ suffer from severe complications. It is difficult to estimate at the time of diagnosis in whom the disease will run an aggressive clinical course. Some studies investigating the role of demographic features on the prognosis of $\mathrm{BD}$ showed that male sex and a younger age of onset (25 years or less) were associated with more severe forms of the disease with vascular, ocular or CNS manifestations. ${ }^{10} 11$ However, there is no simple objective test to predict the prognosis of the disease at the time of diagnosis.

Haemorheological factors have long been implicated in the pathogenesis of vascular lesions in some diseases, such as diabetes mellitus and atherosclerotic heart disease..$^{5612}$ Blood rheology is complex and EA is one of the important haemorheological properties that may create problems at the level of microcirculation. An increase in EA local blood flow and shear rate decrease cause local acidosis and an increase in platelet aggregation that lead to endothelial cell damage. ${ }^{56}$ With this point of view we studied EA rate in $\mathrm{BD}$ to see if it was increased especially in patients with major complications, such as vascular occlusive disease and uveitis. We found that as in the angiopathic forms of diabetic patients, ${ }^{12}$ EA was increased in vascular complications of $\mathrm{BD}$ and 
might be an independent risk factor for an aggressive clinical course. The causative factors for increased EA may be both intrinsic and extrinsic to the erythrocytes. In atherosclerotic diseases, red cell membrane lipid spectrum might change and this may lead to a change in dynamic properties of the red cell membrane. However, we could not find higher chlosterol concentrations in $\mathrm{BD}$ patients. It has long been known that fibrinogen causes formation of rouleaux of erythrocytes. ${ }^{13}$ Each fibrinogen molecule is adsorbed onto the surface of the membrane of adjacent erythrocytes to bridge them, resulting in the formation of erythrocyte aggregates. Fibrinogen also increases blood viscosity, which has a negative effect on blood fluidity. Although we found higher fibrinogen values in $\mathrm{BD}-\mathrm{b}$ when compared with $\mathrm{BD}-\mathrm{a}$ and HC groups, the difference was not significant and they were still in the normal range. Follow up fibrinogen concentrations remained within the normal range in all groups, so it is unlikely that this parameter contributed to higher EA rates. We could not find a definite reason for the increased EA and the exact cause remains to be determined. It is not unusual for EA to be increased in vascular thrombotic disorders without known risk factors. ${ }^{14}$ During follow up, post-M and post-M1 were still higher than $M$ and $\mathrm{M1}$ in BD-a and HC groups $(\mathrm{p}<0.05)$. This might be interpreted as that these patients are still at risk of recurrent vascular-ocular attack.

In conclusion, we suggest that determination of EA is a simple test that can be applied to clinical practice and it might be useful to identify subgroups who are likely candidates for developing vascular complications in BD. Hence, management of factors known to affect blood rheology like stress, cigarette smoking, abnormal lipid profile, and so on, might be of benefit to lower the risk of vascular complications. ${ }^{15}$ Pharmacological approach to the increased EA is a subject of investigation in recent years. $\beta$ Blockers, calcium channel blockers, and ticlopidine are only a few drugs shown to decrease a rise in EA. ${ }^{6}$ However, further and large scale studies are required to elucidate the mechanism(s) of increased EA and its role in the pathogenesis of vascular complications in $\mathrm{BD}$.

There was no financial support for this study.

1 Shimizu T, Erlich GE, Inaba G, Hayashi K. Behçet's disease (Behçet's syndrome). Semin Arthritis Rheum 1979;8:22360.

2 Haim S. Pathogenesis of Behçet's disease. Int J Dermatol 1983;22:101-2.

3 al Dalaan AN, al Ballaa SR, al Janadi MA, Bohlega S, Bahabri S. Association of anti-cardiolipin antibodies with vascular thrombosis and neurological manifestation of Behçet's disease. Clin Rheumatol 1993;12:28-30.

4 Orem A, Değer O, Memiş O, Bahadır S, Ovalı E, Cimşit G. $\mathrm{Lp}$ (a) lipoprotein levels as a predictor of risk for thrombogenic events in patients with Behcet's disease. Ann Rheum Dis 1995;54:726-9.

5 Demiroğlu H, Barışta İ, Dündar S. Erythrocyte aggregability in patients with coronary heart disease. Clinical Hemorheology 1996;16:313-17.

6 Demiroğlu $\mathrm{H}$. The importance of erythrocyte aggregation in blood rheology: considerations on the pathophysiology of blood rheology: considerations on the path
thrombotic disorders. Blood 1997;89:4236.

7 International Study Group for Behçet's Disease. Criteria for diagnosis of Behçet's disease. Lancet 1990;335:1078-80.

8 Rampling MW, Martin G. A comparison of the Myrenne erythrocyte aggregometer with older techniques for estimating erythrocyte aggregation. Clinical Hemorheology 1989;9:41-6.

9 O’Neill TW, Rigby AS, McHugh S. Regional differences in clinical manifestations of Behçet's disease. In: Wechsler B, Godeau P, eds. Behcet's disease.Amsterdam: Excerpta Medica International Congress Series 1037, 1993:159-63.

10 Yazıc1 H, Pazarlı H, Barnes CG, Tüzün Y, Özyazgan Y, Silman A, et al. A controlled trial of azathioprine in Behçet's disease. N Engl J Med 1990;322:281-5.

11 Demiroğlu H, Barıșta İ, Dündar S. Risk factor assessment and prognosis of eye involvement in Behçet's disease in Turkey. Ophthalmology 1997;104:701-5.

12 Demiroğlu H, Gürlek A. Altered red blood cell rheology as a predisposing factor for diabetic nephropathy. Nephron 1998;79:121-2.

13 Evans EA, Brooks DE. Rheology of blood cells. In: Chien S, Dormandy J, Ernst E, Matrai A, eds. Clinical hemorheology. Dordrecht: Martinus Nijhoff Publishers, 1987:73-96.

14 Glacet-Bernard A, Chabanel A, Lelong F, Samama MM, Coscas G. Elevated erythrocyte aggregation in patients with central retinal vein occlusion and without conventional risk factors. Ophthalmology 1994;101:1483-8.

15 Ernst E, Weihmayr T, Schmid M, Baumann M, Matrai A. Cardiovascular risk factors and hemorheology - physical fitness, stress and obesity. Atherosclerosis 1986;59:263-9. 